9 Mandel M A, Gustafsorr Brown C, Savidge B, et al. Molecular characterization of the Arabidopsis floral homeotic gene APET ALA1. Nature, 1992, 360: 273 277

10 Huang H, Tudor M, Su T, et al. DNA binding properties of two Arabidopsis M ADS domain proteins: binding consensus and dimer formation. Plant Cell, 1996, 8: 81 94

11 Angenent G C, Franken J, Busscher M, et al. Petal and stamen formation in petunia is regulated by the homeotic gene fbp1. Plant J, 1993, 4: 101 112

12 Hardenack S, Ye D, Saedler H, et al. Comparison of M ADS box gene expression in developing male and female flow ers of the dioecious plant white campion. Plant Cell, 1994, 6: $1775 \sim 1787$

13 Flanagan C A, Ma H. Spatially and temporally regulated expression of the MADS-box gene AGL2 in wild type and mutant Arabidopsis flowers. Plant Mol Biol, 1994, 26: 581 595

14 Jofuku K D, Den Boer B G W, Van Montagu M, et al. Control of Arabidopsis flow er and seed development by the homeotic gene APETALA2. Plant Cell, 1994, 6: $1211 \sim 1225$

\title{
水蛭机械感觉通道间突触联系对机械 感觉信息传入的调控
}

\author{
黄 欣 黄立军 张人骥* \\ ( 北京大学生命科学学院生理学及生物物理学系, 北京 100871.* 联系人)
}

摘要 感觉系统中一种特定感觉型(Modality) 或者亚感觉型的感觉信息传递机制被称为一种感觉通道( Sensory channel), 包括感受器, 感觉传入通路及处理此种感觉信息以产生相应知觉的中枢部分 ${ }^{[1,2]}$. 一种感觉通 道可由一定的适宜刺激激活, 经相应的感受器换能后传入中枢. 感受器的特性很大程度上决定了此种感觉型 或亚感觉型的性质。然而, 一种感觉型的性质除了受相应感觉通道的感受器特性影响外, 是否还由其他因素 决定? 是否存在某种门控 (Gating) 机制对基于不同感觉通道的感觉信息的传入进行选择呢? 通过对水蛭机 械感觉型间机能关系的研究回答上述问题.

\section{关键词 水蛭 机械感受 感觉通道 感觉型门控机制 中枢神经系统}

水蛭神经系统由神经节链组成. 其体神经节中机械感受触 $(T) 、$ 压 $(P)$ 、伤害性 $(N)$ 神经元 均已被标定. 神经节每侧有 3 种机械感觉型共 7 个机械感受神经元, 分别为 3 个 $\mathrm{T}$ 细胞( $\mathrm{T} 1$, T2.T3). 2 个 P 细胞 (P1, P2) 和 2 个 $N$ 细胞 (N1.N2). 每个细胞有其特定的体表感受野 区 $^{[3]}$. 因而, 水蛭机械感受神经元既反映外周的特性, 也具备中枢的性质 ${ }^{[3,4]}$. 水蛭神经系统是研究 感觉通道间相互关系的适宜标本. 在欧洲医蛭上显示过不同感觉型感受细胞间的相互关 系 $^{[5 \sim 7]}$. 迄今, 未见有关于水蛭的机械感觉型性质与 3 种机械感觉通道间机能相关的报道; 也 未见提出决定水蛭不同机械感觉信息的选择性传入的机制.

\section{1 材料与方法}

取材：中华宽体金线蛭 Whitmania pigra, 采自北京郊区及河北沧县. 取体长 6 $12 \mathrm{~cm}$ 的成体动物进行实验.

实验标本: 用 $15 \%$ 酒精麻醉至肌肉松弛. 制备单个神经节及神经节-体壁表皮标本 ${ }^{[6]}$. 
后者的制备需保留一侧神经根完好, 并取下该侧从腹中线到背中线的表皮及体壁 ${ }^{8]}$.

电生理学记录: 采用细胞内记录. 玻璃微电极内充 $4 \mathrm{~mol} / \mathrm{L} \mathrm{KAc}$ 溶液, 电阻 $20 \sim 40 \mathrm{M} \Omega$. 刺激方式有两种: 给予细胞内注入电流 (1 5 nA) 或通过压电晶体, 将电压转换为玻璃棒位移 直接刺激皮肤. 用 3 根微电极同时记录. 信号经 WPI-KS700, WPI-M 4A, Axoclamp 2B 放大 器放大, 经电平调节和 TL-1 DMA 接口, 由计算机采样.

\section{2 实验结果}

2.1 不同机械感觉型间的相互关系

用 3 根微电极同时胞内记录单个体神经节一侧 $\mathrm{T}, \mathrm{P}, \mathrm{N}$ 细胞的活动. $\mathrm{T}$ 细胞内通入去极 化电流未见在 $\mathrm{P}$ 细胞及 $\mathrm{N}$ 细胞引起相应反应. 刺激 $\mathrm{N}$ 细胞诱发单个动作电位可在 $\mathrm{P}$ 细胞引 起幅度约为 $0.4 \mathrm{mV}$ 的兴奋性突触后电位 $(\mathrm{epsp})$. 刺激 $\mathrm{P}$ 细胞, 在 $\mathrm{N}$ 细胞上未见反应; 在 $\mathrm{T}$ 细胞上引起的 epsp 及稍后出现的持续时间可长达 800 $1000 \mathrm{~ms}$, 幅度可达 $8 \mathrm{mV}$ 的多个抑制 性突触后电位 (ipsp), 见图 1(a).

$\mathrm{P}$ 细胞在 T 细胞上产生短延时的 epsp 和相对长延时的 ipsp, 图 1(b). 未见 P 细胞的多个 动作电位发放对 $\mathrm{T}$ 细胞引起的 epsp 有易化现象. 加入高 $\mathrm{Mg}^{2+}$ 溶液 $2 \mathrm{~min}$ 后, ipsp 消失, 而 epsp 的幅度、时程均无改变, 图 1(c). 在 $\mathrm{T}$ 细胞通入超极化电流, 未见在 P 细胞产生相应变 化, 图 1(d). 提示: $\mathrm{P}$ 细胞到 $\mathrm{T}$ 细胞的兴奋性联系由整流型电突触传递; 抑制性联系由化学突 触传递.

\section{$2.2 P$ 细胞对 $\mathbf{T}$ 细胞的兴奋和抑制}

同时记录一个体神经节一侧 2 个 $\mathrm{T}$ 细胞和 1 个 $\mathrm{P}$ 细胞的活动, 在 $\mathrm{T}$ 细胞上可见自发的 ipsp; ipsp 大致同步, 但也有不同步成分, 图 2(a) 作为对照. 给予 $\mathrm{P}$ 细胞胞内刺激, 可见在 $\mathrm{T}$ 细胞上产生的 epsp, 以及频率及幅度均明显超过对照组的 ipsp, 图 2(b).

刺激 $\mathrm{P}$ 细胞, 同时记录同侧 $\mathrm{T} 1, \mathrm{~T} 3$ 细胞活动, 或记录 $\mathrm{T} 2, \mathrm{~T} 3$ 细胞的活动, 可见在任 2 个 $\mathrm{T}$ 细胞上产生的 ipsp 基本同步, 图 2(c) 及图 2(d). 由此提示, 刺激 $\mathrm{P}$ 细胞在同侧的全部 3 个 $\mathrm{T}$ 细胞上产生基本同步的抑制作用. 刺激 $\mathrm{P} 1, \mathrm{P} 2$ 细胞结果相同. $\mathrm{T}$ 细胞与被刺激的 $\mathrm{P}$ 细胞有 部分共同的体表感受野支配区域, 未见 $\mathrm{P}$ 细胞的发放对与该 $\mathrm{P}$ 细胞具有对应感受野的 $\mathrm{T}$ 细胞 的兴奋和抑制作用超过对具有非对应感受野的 $\mathrm{T}$ 细胞的作用; 亦即, 未表现出感受野部位的 优势对应关系. $\mathrm{P}$ 细胞对 $\mathrm{T}$ 细胞的兴奋和抑制作用表现为压力觉和触觉两种机械感觉型间的 一般关系.

控制胞内刺激 $\mathrm{P}$ 细胞的强度, 只产生单动作电位反应, 可见 $\mathrm{P}$ 细胞的一个动作电位即能 在 $\mathrm{T}$ 细胞上产生明显的 ipsp. 随着刺激强度的增加, $\mathrm{P}$ 细胞的多个动作电位发放, 在 $\mathrm{T}$ 细胞上 引起的 ipsp 的频率明显提高. $\mathrm{P}$ 细胞兴奋在 $\mathrm{T}$ 细胞引起的 epsp 有时能引发动作电位.

对神经节-体壁表皮标本施加刺激, 当机械刺激强度达到激活 P 细胞感受范围时, 可同时 在本节和邻节同侧的 $\mathrm{T}$ 细胞上见到 ipsp. 本节和邻节 $\mathrm{T}$ 细胞间的 ipsp 有同步成分, 也有不同 步成分.

\section{3 讨论}

上述结果提示: 机械感觉型的性质并非仅由该感觉通道的机械感受器特性决定; 不同感觉 通道之间在中枢的相互作用对决定感觉型的性质有重要贡献. 触觉感觉型具有适应快, 对刺 


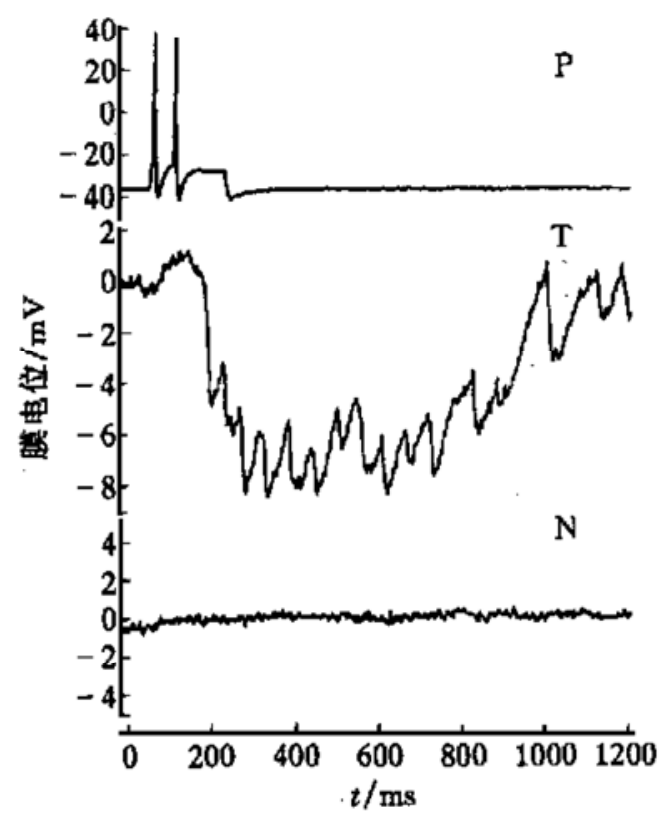

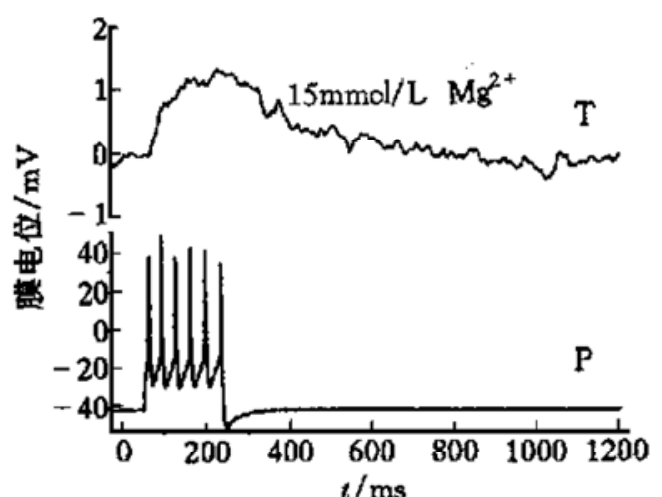

(c)

(d)

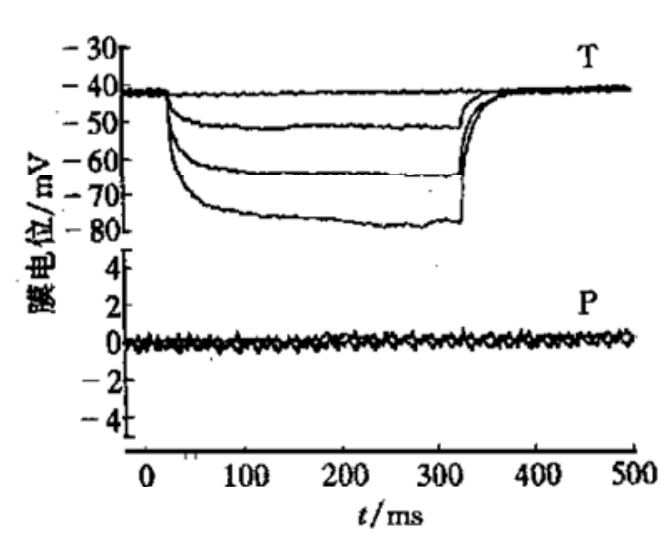

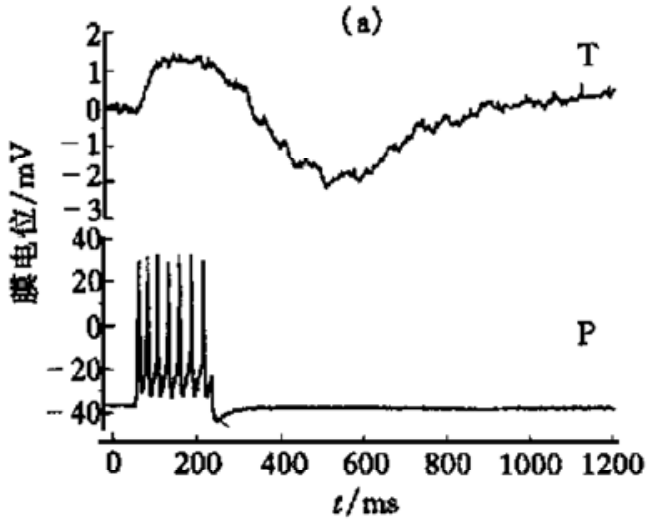

(b)

图 1 不同机械感觉型间的相互关系

(a) 胞内刺激 $\mathrm{P}$ 细胞, 同时在同侧的 $\mathrm{T}$ 细胞和 $\mathrm{N}$ 细胞胞内记录的结果; ( b) 胞内刺激 $\mathrm{P}$ 细胞, 在同侧 $\mathrm{T}$ 细胞胞内记 录. 结果为 6 次实验的平均; (c) 加 $15 \mathrm{mmol} / \mathrm{L}$ 高 $\mathrm{Mg}^{2+}$ 溶液, 胞内刺激 $\mathrm{P}$ 细胞, 在同侧 $\mathrm{T}$ 细胞胞内记录. 结果为 6 次实验平均; (d) $\mathrm{T}$ 细胞胞内通以超极化电流, 在同侧 $\mathrm{P}$ 细胞胞内记录的结果

激强度的变化敏感的特性. 对应于机械刺激发生变化达到压力觉阈值, $\mathrm{P}$ 细胞开始兴奋而发 放时, 经电突触在触觉 $\mathrm{T}$ 细胞上引发的短延时 epsp, 甚至动作电位使 $\mathrm{T}$ 细胞的发放频率增加, 这增强了 $\mathrm{T}$ 细胞对强度变化的检测. 触觉对强度的变化敏感的相位特性由于压力觉通道对 触觉通道的兴奋作用而增强.

多个感觉通道的并行存在是生物为获得感觉的高灵敏度和宽动态范围而采取的策略. 对 应于并行感觉通道间相互重叠的情况, 合理选择最适感觉通道并排除其他通道的干扰, 对于感 觉信息的传入和在中枢的明确阐述具有重要意义. $\mathrm{P}$ 细胞的兴奋在 $\mathrm{T}$ 细胞上引起的 ipsp 终 止了 $\mathrm{T}$ 细胞的兴奋, 并使其处于一段相当长时间的抑制状态. 而 $\mathrm{P}$ 细胞到 $\mathrm{T}$ 细胞的整流型电 突触连接使 $\mathrm{T}$ 细胞的超极化对突触前 $\mathrm{P}$ 细胞没有影响. 这如同存在一个感觉通道的转换开 关, 在压力感觉通道被激活时, 通过此“开关” 关闭了触觉感觉通道, 使动物只明确地感受到一 种刺激强度的信息. 感觉通道间的相互作用为感觉信息的选择传入提供了门控机制. 与压力 


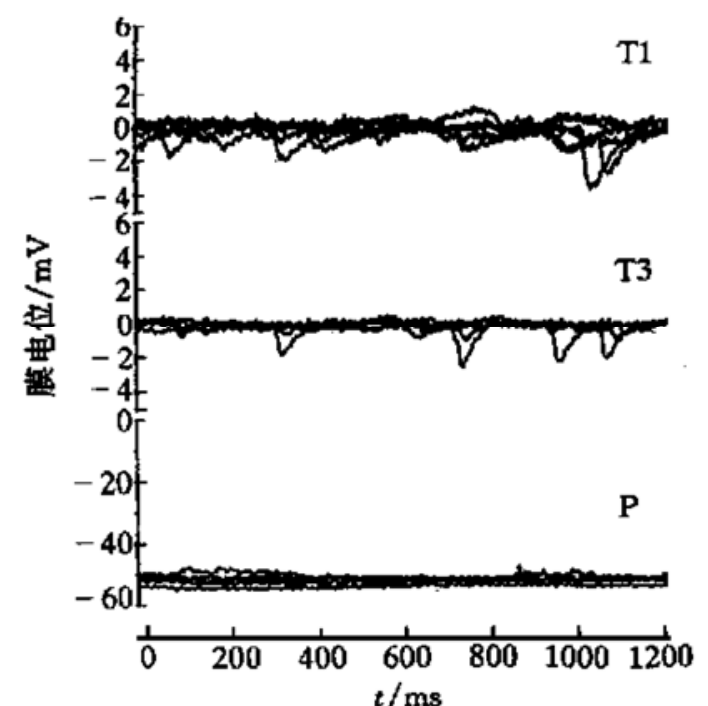

(a)

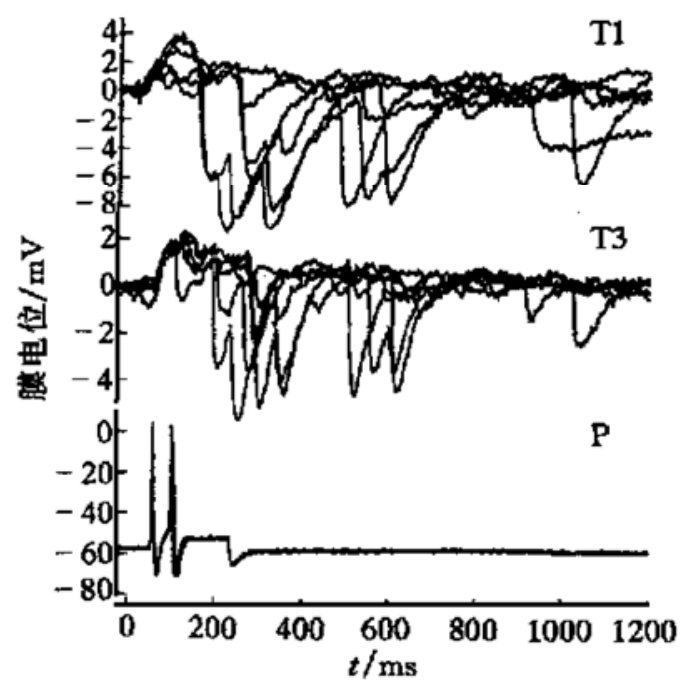

(b)

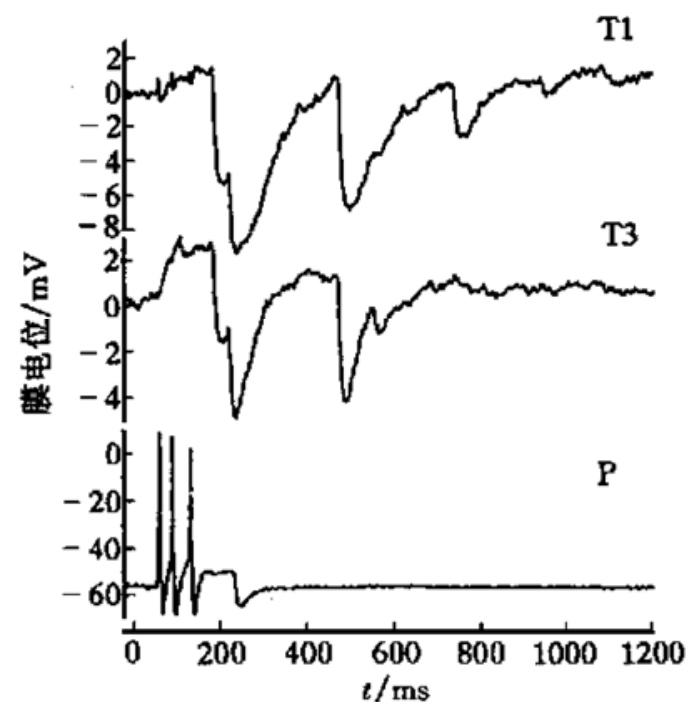

(c)

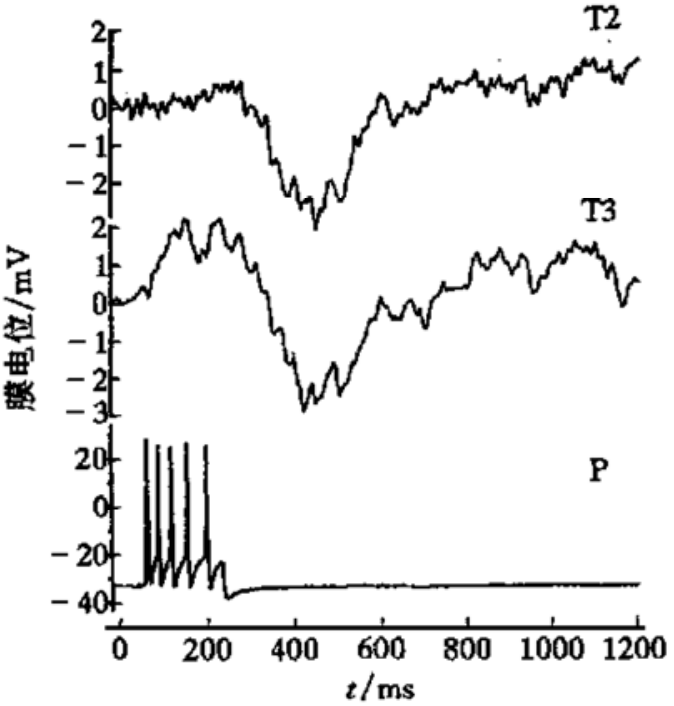

(d)

图 $2 \quad \mathrm{P}$ 细胞的兴奋对 $\mathrm{T}$ 细胞有兴奋和抑制作用

(a) 不刺激 $\mathrm{P}$ 细胞, 同时在同侧的 $\mathrm{T} 1, \mathrm{~T} 3$ 细胞胞内记录. 结果为 6 次记录的迭加. 可见在 $\mathrm{T}$ 细胞上的自发 ipsp. 注意到有同步和异步成分的存在; (b) 胞内刺激 P 细胞, 在同侧的 T 1, T3 细胞上见到明显的 epsp 和 ipsp. ipsp 的 频率和幅度明显超过对照组. 结果为 6 次记录的迭加; ( c) 胞内刺激 $\mathrm{P}$ 细胞, 同时在同侧的 $\mathrm{T} 1, \mathrm{~T} 3$ 细胞胞内记录 的结果. $\mathrm{T} 1, \mathrm{~T} 3$ 细胞上的 ipsp 基本同步, 但也可见个别不同步成分; (d) 胞内刺激 $\mathrm{P}$ 细胞, 同时在同侧的 $\mathrm{T} 2, \mathrm{~T} 3$ 细 胞胞内记录的结果. T2, T3 细胞上的 ipsp 基本同步, 但也可见个别不同步成分

觉和触觉感觉通道间的关系不同, 伤害觉通道的激活并不抑制压力觉通道, 水蛭同时保持对刺 激强度与伤害性的感受能力. 本文结果见图 3.

$\mathrm{P}$ 细胞在同节同侧 3 个 $\mathrm{T}$ 细胞上引起基本同步的 ipsp, 推测 $\mathrm{P}$ 细胞兴奋了一个抑制单元. $\mathrm{P}$ 细胞的少数 ( 2 个或 3 个) 动作电位的发放能够在 $\mathrm{T}$ 细胞上引发持续时间可长达 $100 \mathrm{~ms}$ 的 多个ipsp, 提示抑制单元由多个抑制性中间神经元组成, 它们之间可能存在较强的兴奋性联 系. 正反馈的存在可使经 P 细胞兴奋激活的抑制单元的串发放持续较长时间. 不同步 ipsp 的存在以及邻节同步 ipsp 间存在时差也可能与抑制单元内多个抑制性中间神经元的活动有 关. 


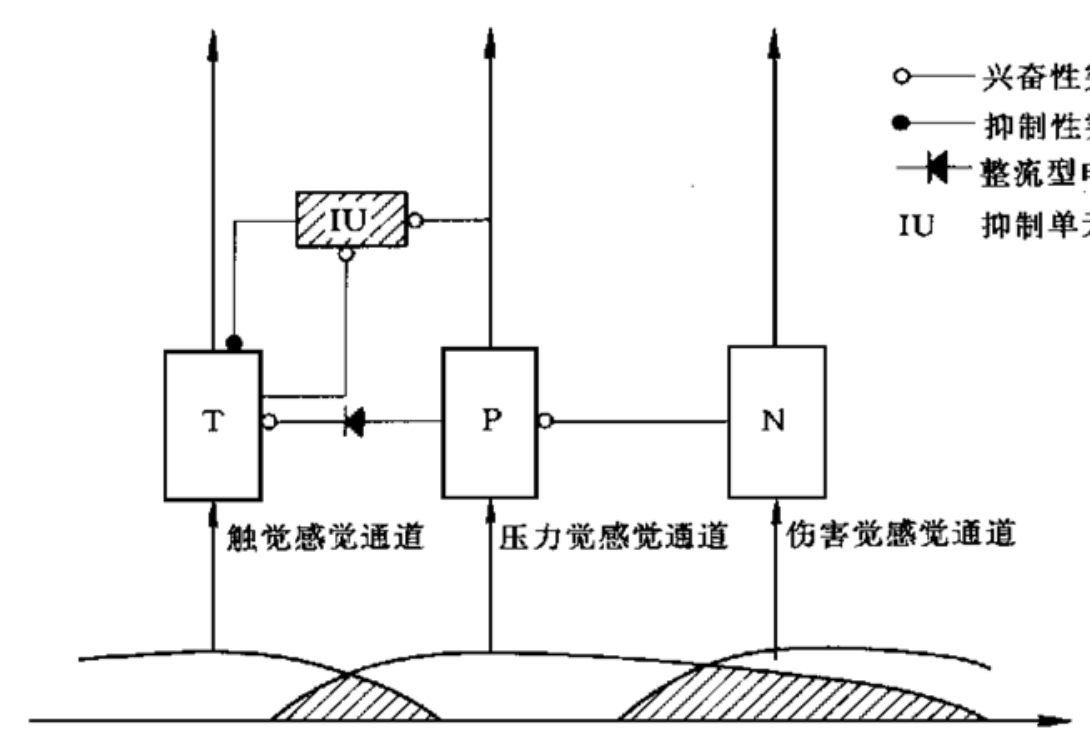

㧈械刺激强度(按强度分列 3 种感知型)

图 3 水蛭不同机械感觉型间相互关系示意图

图下方曲线示意不同机械感觉通道对机械刺激强度的感受范围. 曲线间相交的阴影部分表明处于此强度范围内 的机械刺激可以同时激活两种感觉通道. 激活压力觉感觉通道可以通过抑制单元抑制触觉感觉通道, 控制感觉信 息从特定的感觉通道传入. 与压力觉通道不同, 伤害觉通道传递的是伤害性信息, 从此意义上讲, 这两者不是同一 性质的并行感觉通道. 假定水蛭的中枢神经系统需要同时了解刺激的强度和刺激的伤害程度, 这可以解释为什么 激活伤害觉通道对压力觉通道并无抑制作用

致谢 本工作为国家科委“攀登”计划支持课题.

\section{参 考 文 献}

1 Braddick O, Campbell F W, Atkinson J. Channels in vision: basic aspects. In: Held R, Leibow itz H W, Teuber H L eds. Handbook of Sensory Physiology, Vol. 8, Perception, Berlin_New York: Springer Verlag, 1978. 9 12

2 Willis W D, Coggeshall R E. Sensory M echanisms of the Spinal Cord, 2nd ed, Chap 10. New York: Plenum Press, 1991. 401

3 Nicholls J G, Baylor D A. Specific modalities and receptive fields of sensory neurons in CNS of the leech. J Neurophysiol, 1968, 31: 740 756

4 Blackshaw A M. Morphology and distribution of touch cell terminals in the skin of the leech. J Physiol, 1981, 320: 219 228

5 Baylor D A, Nicholls J G. Chemical and electrical synaptic connexions between cutaneous mechanoreceptor neurones in the central nervous system of the leech. J Physiol, 1969, 203: 591 609

6 Muller K J, Nicholls J G, Stent G S. Neurobiology of the Leech. New York: Cold Spring Harbor Laboratory Press, 1981. 249 275

7 Nicholls J G, Wallace B G. Modulation of transmission at an inhibitory synapse in the central nervous system of the leech. J Physiol, 1978, 281: 157 170

8 张 帆, 张人瀷. 水蛭中枢神经元节段再支配的方位识别与低阻贯通调节. 中国科学, B 辑, 1992(12): $1287 \sim 1294$ 